\title{
Evaluación interanual de las estrategias regenerativas de la especie exótica invasora Gleditsia triacanthos en relación a la nativa Acacia aroma en el bosque chaqueño serrano de Córdoba (Argentina)
}

\author{
Interannual evaluation of the regenerative strategies of the exotic invasive species Gleditsia triacanthos \\ compared with the native Acacia aroma in the Chaco Serrano Woodland of Cordoba (Argentina)
}

\author{
Ana Elisa Ferreras a*, Guillermo Funes a,b, Leonardo Galetto a,b

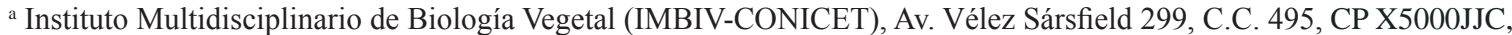 \\ Córdoba, Argentina, tel.: 54-351- 5353800 int. 30053, fax: 54-351-4331056, aferreras@imbiv.unc.edu.ar \\ b Universidad Nacional de Córdoba, Facultad de Ciencias Exactas, Físicas y Naturales, Escuela de Biología, Córdoba, Argentina.
}

\begin{abstract}
SUMMARY
It has been proposed that exotic species, compared with native ones, differed in certain characteristics that may favor their expansion. The regenerative characteristics of exotic species may vary among reproductive seasons, modifying the patterns observed contrasted with native species and hence influencing their expansion velocity. In this work, fruit production, seed density in the soil and seedlings density around the con-specifics and in the study sites were compared between the exotic invasive Gleditsia triacanthos and the native Acacia aroma. The study was developed in at least two reproductive seasons, in areas of the Chaco Serrano Woodland of Córdoba (Argentina). In each season, the number of fruits was counted. Around the con-specifics of each species it was obtained: a) the density of seeds in the soil (in two different moments per year) and b) the density of seedlings. In addition, the density of seedlings distributed in the study sites was registered. In two of the three reproductive seasons analyzed, fruit production was higher in the exotic invasive species. In both seasons the density of seeds in the seed bank and of seedlings around the focal individuals was higher in the exotic species. The density of seedlings in the study sites did not vary between species in any of the seasons. The regenerative characteristics observed in $G$. triacanthos and the consistency in their response in different reproductive seasons suggest a rapid expansion of the species in the region.
\end{abstract}

Keywords: seed bank, temporal changes, seedling density, fruit production.

\section{RESUMEN}

Se ha propuesto que las especies exóticas, en relación a las nativas, difieren en características o estrategias que favorecerían su expansión. Las características regenerativas de las plantas exóticas pueden variar entre temporadas reproductivas, modificando los patrones con respecto a las especies nativas e influyendo en la velocidad de expansión. En este trabajo, en el bosque chaqueño serrano de Córdoba (Argentina), se comparó en al menos dos temporadas reproductivas la producción de frutos, la densidad de semillas en el banco y la densidad de plántulas alrededor de los adultos y distribuidas en el área de estudio entre la especie exótica invasora Gleditsia triacanthos y la nativa Acacia aroma. En cada temporada de muestreo se contó el número de frutos por individuo y se obtuvo alrededor de los adultos de cada especie: a) la densidad de semillas en el banco (en dos momentos del año) y b) la densidad plántulas. Además, se registró la densidad de plántulas de ambas especies dispersas en los sitios de estudio. En dos de las tres temporadas analizadas la producción de frutos fue mayor en la especie exótica. La densidad de semillas en el banco y de plántulas alrededor de los adultos fue mayor en la especie exótica en las dos temporadas registradas. La densidad de plántulas en los sitios no varío entre las especies en ninguna de las temporadas. Las características regenerativas de G. triacanthos y la consistencia observada en las mismas en las distintas temporadas sugerirían una rápida capacidad de expansión en la región.

Palabras clave: banco de semillas, cambios temporales, densidad de plántulas, producción de frutos.

\section{INTRODUCCIÓN}

Uno de los problemas ecológicos y económicos contemporáneos más importantes lo constituyen las especies exóticas invasoras (Richardson y Rejmánek 2004, Pyšek y Richardson 2007, Richardson y Rejmánek 2011). Las mismas son un foco de atención desde la perspectiva científica, política, económica y social, principalmente debido a las modificaciones que generan en los ambientes en que se introducen (Vilà et al. 2010). En este sentido, en algunos ambientes invadidos se ha generado la necesidad de urgentes estrategias de manejo, para lo cual es necesario disponer de conocimiento sobre la ecología de estas especies.

Una aproximación al conocimiento de las características o estrategias de las especies exóticas es a través de la comparación con especies nativas (Pyšek y Richardson 
2007). Dicho enfoque permite establecer una línea de base respecto de aquellos caracteres que serían responsables de los procesos de invasión (Pyšek y Richardson 2007); por ejemplo, si una especie exótica presenta mayor tasa de crecimiento que nativas con similares características ecológicas, es posible que esta característica esté contribuyendo a su expansión en ese sistema. Asimismo, este enfoque presenta un sólido desarrollo teórico ya que algunas de las ideas más importantes que intentan explicar los procesos de invasión se basan en comparaciones con especies nativas -i.e., hipótesis de naturalización de Darwin (Strauss et al. 2006). Además, permite diseñar protocolos sobre los riesgos de establecimiento e invasión de una especie (Maguire 2004). En este contexto, se ha propuesto que las plantas exóticas invasoras presentarían una o más características o estrategias distintivas que les facilitarían la expansión en las comunidades donde se establecen -e.g., alta producción de semillas, mayores áreas foliares, etc. (Pyšek y Richardson 2007). Por otra parte, el periodo regenerativo es clave en la vida de una especie, contribuyendo a determinar la densidad final de las poblaciones (Sakai et al. 2001) y los patrones de distribución espacial, en pequeña y gran escala -i.e., su distribución tanto en la escala local como en los límites de su rango geográfico (referencias en Martin y Canham 2010). En consecuencia, se ha resaltado la importancia de estudiar este periodo del ciclo de vida para conocer el potencial invasor de una planta, ya que influiría en su capacidad para colonizar, establecerse y expandirse (Pairon et al. 2006, Martin y Canham 2010).

Dentro del cuerpo de estudios que comparan estrategias regenerativas en especies exóticas, pocos han considerado la variabilidad temporal que dichas estrategias podrían presentar. Sin embargo, evaluar estas variaciones permitiría conocer si ciertos caracteres o estrategias pueden cambiar entre temporadas reproductivas y permitiría estimar la velocidad de invasión (Pairon et al. 2006, Martin y Canham 2010). Por ejemplo, Pairon et al. (2006), en Bélgica, encontraron grandes variaciones en la producción de frutos en dos años consecutivos para la exótica invasora Prunus serotina Ehrh. Así, si la especie exótica presenta en relación a la nativa estrategias que favorecen su invasión en la mayoría de las temporadas reproductivas (por ejemplo, mayor producción de frutos), su velocidad de invasión sería mayor que si dicho comportamiento se presentara sólo esporádicamente (Hastings et al. 2005). Además, una gran variabilidad interanual en las estrategias regenerativas contribuiría a explicar la inconsistencia que suele encontrarse en el cuerpo de caracteres que describen a las especies exóticas respecto a las nativas (Pyšek y Richardson 2007). Por otro lado, el conocimiento del comportamiento de las estrategias regenerativas entre temporadas reproductivas podría tener importantes consecuencias para planear estrategias de manejo -i.e., temporadas en que las especies presenten ciertas características, como baja densidad de plántulas, podrían ser más favorables para aplicar estrategias de manejo.
Trabajos previos sugieren que la producción de frutos puede ser muy variable en las distintas temporadas, fenómeno denominado "semillación masiva” (del inglés "mast seeding”), que implica, en plantas perennes, la alternancia de años con alta producción de frutos/semillas con años de baja producción (Kelly y Sork 2002). El mismo podría ser disparado, en el corto plazo, por condiciones ambientales favorables o por una mayor disponibilidad de recursos para la reproducción, las cuales podrían generar, respectivamente, una mayor disponibilidad de polinizadores o una mayor producción de flores (Kelly y Sork 2002). En este sentido, se ha encontrado que las especies nativas presentan mayores fallas en la reproducción en relación a las exóticas (Martin y Canham 2010) y que especies con mayores niveles de invasividad registran periodos más cortos entre años de alta fructificación (Richardson y Rejmánek 2004). Asimismo, las variaciones en la producción de frutos repercutirían en otras variables del ciclo reproductivo de las especies, pudiendo generar, por ejemplo, variaciones en la densidad de semillas del banco o de plántulas establecidas. A su vez, la variabilidad interanual en estas últimas variables regenerativas podría deberse a otros factores como cambios en la germinabilidad de las semillas, variaciones anuales en la comunidad de herbívoros o en hongos, microbios y otros depredadores que se encuentren en el suelo (Rudgers y Hoeksema 2003, Krinke et al. 2005). Además, factores abióticos, lluvias o fuegos particulares de cada temporada, podrían influir en la densidad de semillas o plántulas (Krinke et al. 2005).

Sudamérica es considerada una de las regiones más afectadas por especies exóticas invasoras (Fonseca et al. 2013). En Argentina, la alta abundancia de algunas especies exóticas invasoras, constituye uno de los problemas ecológicos más importantes (Chaneton et al. 2004). Gleditsia triacanthos L. -Fabaceae- es una especie nativa de Norte América, que ha sido descripta como invasora en varias regiones del mundo (Richardson y Rejmánek 2011). En Argentina, fue introducida a principios del siglo XIX y actualmente está invadiendo distintas eco-regiones del país (de Viana y Colombo Speroni 2000, Zalba y Villamil 2002, Chaneton et al. 2004). En el Chaco Serrano de Córdoba, G. triacanthos se está expandiendo con gran velocidad en comunidades donde habitan otras leguminosas nativas aunque no existen congéneres nativos de esta especie en dicha región (Giorgis et al. 2011). Acacia aroma Gillies ex Hook. et Arn. -Fabaceae- es una especie nativa con distribución sudamericana (Ebinger et al. 2000). Dentro del grupo de las Fabaceas nativas de esta región, A. aroma presenta caracteres interesantes para ser comparada con G. triacanthos en términos regenerativos, ya que ambas tienen una fenología de la floración y fructificación generalmente superpuesta, siendo esta última bastante prolongada, y frutos indehiscentes con similares mecanismos de dispersión (Ferreras y Galetto 2010). Además, en esta área de estudio ambas especies crecen juntas, siendo $A$. aroma una de las Fabaceae más comunes de encontrar. Por 
otro lado, la bibliografía sugiere que es necesario aumentar el cuerpo de estudios enfocado en especies de hábito arbóreo, dado que su impacto en la comunidad posiblemente sea mayor que aquellas con ciclo de vida corto (Cordell et al. 2009). En un estudio previo enfocado en estas especies se evaluó la producción de frutos, el banco de semillas y la densidad de plántulas en la temporada reproductiva 2007 y en una escala espacial más reducida en relación al presente trabajo (Ferreras y Galetto 2010). En dicho estudio se encontró mayor abundancia de la especie exótica para el banco de semillas tomado con anterioridad al periodo de dispersión, mayor densidad de plántulas alrededor de los adultos de $G$. triacanthos, y valores similares para la producción de frutos, para la densidad del banco de semillas luego de la dispersión y plántulas en el área de estudio (Ferreras y Galetto 2010). A raíz de dicho estudio y del escaso conocimiento de la variabilidad interanual de las estrategias regenerativas tanto para especies exóticas como nativas (Martin y Canham 2010) surgió la necesidad de evaluar si los patrones encontrados entre estas especies en una temporada se mantenían en sucesivas temporadas reproductivas.

En resumen, trabajos previos indican que las especies exóticas tendrían ciertas características que favorecen su invasión (Pyšek y Richardson 2007). Dichas características pueden variar entre temporadas reproductivas (Krinke et al. 2005, Martin y Canham 2010) influyendo en la velocidad de invasión. En particular, en especies con alta capacidad de invasión como G. triacanthos (de Viana y Colombo Speroni 2000, Zalba y Villamil 2002, Chaneton et al. 2004), se espera que las diferencias en relación a la especie nativa sean consistentes entre años, presentando en todas las temporadas características que favorezcan su invasibilidad (mayor producción de frutos, mayor densidad de semillas en el banco y mayor densidad de plántulas). En consecuencia, la velocidad de expansión de la especie exótica sería rápida y podría conducir a una pronta conversión del bosque nativo en un bosque mono-específico de G. triacanthos. El objetivo de este trabajo es comparar la producción de frutos, la densidad de semillas en el banco y la densidad de plántulas alrededor de los adultos de cada especie y en los sitios, entre la especie exótica invasora $G$. triacanthos y la nativa A. aroma en dos temporadas reproductivas (2008 y 2009) en el bosque chaqueño serrano de Córdoba, Argentina.

\section{MÉTODOS}

Área de estudio y diseño general del estudio. El estudio se desarrolló en tres sitios de bosque chaqueño serrano ubicados en las localidades de La Rancherita (31 ${ }^{\circ} 45^{\prime} 34,58^{\prime}$ ' S, 64²7'34,19”' O), La Serranita (3144'18,01'” S,

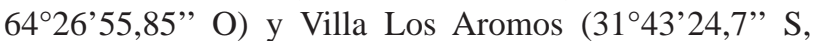
64²6’30,4”' O), Departamento Santa María, Córdoba, Argentina. La precipitación promedio anual es de 734 mm, concentrada en el periodo septiembre-abril (De Fina 1992).
La temperatura media de verano es de $24^{\circ} \mathrm{C}$ y la de invierno $10^{\circ} \mathrm{C}$ (De Fina 1992). En la estructura de la vegetación se distinguen tres estratos: arbóreo, generalmente abierto, arbustivo y herbáceo. La vegetación de leñosas nativas en el área estudiada consiste principalmente en Lithraea molleoides (Vell.) Engl., Zanthoxylum coco Gillies ex Hook. f. et Arn., Ruprechtia apetala Wedd., Celtis spp., y dentro de las leguminosas más importantes se encuentran Prosopis alba Griseb., Acacia caven (Molina) Molina y Acacia aroma. También se destacan especies exóticas invasoras como Gleditsia triacanthos, Ulmus spp., Ligustrum lucidum W. T. Aiton y Pyracantha angustifolia (Franch.) C. K. Schneid. (Giorgis et al. 2011).

Los tres sitios donde se realizó el estudio pueden ser considerados en un estado incipiente de invasión -i.e., presentan individuos adultos reproductivos de G. triacanthos, en general aislados. Además, estos sitios resultan interesantes de abordar por su proximidad a localidades donde G. triacanthos se encuentra en un estado de invasión más avanzado, formando bosques mono-específicos. Se consideraron siete individuos de cada especie en cada sitio -i.e., total de 21 individuos por especie- a excepción del muestreo de banco de semillas en el que para la especie exótica, en el sitio de Los Aromos, se pudieron obtener datos de sólo cinco individuos. Todas las variables fueron evaluadas en dos temporadas reproductivas (2008 y 2009), a excepción de la producción de frutos que también se evaluó en la temporada 2010. Las variables regenerativas fueron evaluadas sobre los mismos individuos en ambas temporadas. La selección de los individuos se hizo al azar dentro de un grupo de individuos en edad reproductiva.

Producción de frutos. Se realizó un conteo completo del número total de frutos de los individuos de cada especie. En los casos en que la producción de frutos era muy alta, se la estimó a través de conteos del número de frutos de una a tres ramas representativas del individuo -i.e., si el individuo presentaba un producción heterogénea (muchos frutos concentrados en algunas ramas, y otras ramas con pocos frutos) se eligieron ramas modelo de las distintas situaciones-, y luego dicho número se multiplicó por el número total de ramas con frutos de dicho individuo (Ferreras y Galetto 2010).

Banco de semillas. Para evaluar la persistencia de las semillas y su densidad en el banco se extrajeron muestras de suelo debajo de los individuos focales de cada especie. En cada temporada, las muestras de suelo fueron tomadas en dos momentos distintos. El primer momento fue luego de finalizada la dispersión, comenzando el periodo de lluvias -octubre-noviembre- ("banco de semillas luego de la dispersión”) y el segundo momento previo a la nueva temporada de dispersión -mayo-junio- ("banco de semillas previo al nuevo periodo de dispersión”). Esto permitió evaluar si las plantas presentan semillas viables en el suelo a lo largo de todo el año. 
Bajo cada individuo se colectaron tres muestras de suelo de 20 x 20 x $4 \mathrm{~cm}$ que fueron tamizadas en laboratorio. Se consideraron solo aquellas semillas que se encontraban sin la cubierta del fruto. Posteriormente se colocaron a germinar en cápsulas de Petri (cada individuo en una cápsula independiente), en cámaras de germinación bajo condiciones de luz y temperatura controladas (fotoperiodo de $12 \mathrm{~h}$, densidad de la luz próxima a $40 \mu \mathrm{mol} \mathrm{m} \mathrm{m}^{-2} \mathrm{~s}^{-1}$, temperatura $25^{\circ} \mathrm{C}$ ). Se contabilizó la cantidad de semillas germinadas, considerándose germinadas aquellas semillas en que había emergencia de $2 \mathrm{~mm}$ de la radícula. Aquellas semillas que, luego de 30 días, no germinaron, fueron escarificadas mecánicamente con lija y colocadas nuevamente en las cámaras de germinación (este proceso se repitió hasta que todas las semillas hubieran germinado o pudieran ser claramente identificadas como muertas).

Densidad de plántulas. El reclutamiento de plántulas fue estimado a través de dos aproximaciones: a) debido a que se ha observado que estas especies suelen presentar un crecimiento agregado, se realizaron muestreos alrededor de los adultos de ambas especies, b) dado que ambas especies presentan dispersión por vertebrados (Ferreras y Galetto 2010), quienes trasladarían las semillas a distintas partes de los sitios, se estimó la densidad de plántulas en el área de estudio. Los muestreos fueron realizados en diciembre de cada temporada, ya que durante este mes las semillas de ambas especies ya habían estado sujetas a un periodo suficientemente prolongado de oportunidades para germinar -i.e., lluvias y temperaturas necesarias. Se consideraron como plántulas a aquellas que tenían hasta $30 \mathrm{~cm}$ de altura. Este criterio se estableció a partir de la altura máxima de plántulas crecidas en laboratorio a los 80 días desde su germinación ${ }^{1}$ (este lapso de tiempo representa aproximadamente el periodo transcurrido desde el inicio de las lluvias al momento de muestreo de las plántulas).

Densidad de plántulas alrededor de los individuos adultos. Para comparar la densidad de plántulas alrededor de los adultos de cada especie se dispusieron parcelas ubicadas en un radio de 10 metros alrededor de los individuos adultos de cada especie -i.e., individuos focales. Para esto, en cada temporada se utilizaron dos transectas de $10 \mathrm{~m}$ de largo partiendo de los individuos focales. En cada una de ellas se estableció una parcela rectangular de 2 x $1 \mathrm{~m}$ $\left(2 \mathrm{~m}^{2}\right)$ al inicio del transecto, a los cinco y a los diez metros de la planta focal, donde se registraron todas las plántulas existentes de la especie focal (un total de $12 \mathrm{~m}^{2}$ muestreados por individuo, por temporada).

Densidad de plántulas en el área de estudio. Para determinar la densidad de plántulas se establecieron 24 parcelas en cada uno de los sitios de muestreo (total 72 parcelas por

Melgar et al. Instituto Multidisciplinario de Biología Vegetal, CONICET, Universidad Nacional de Córdoba, Argentina. Datos no publicados.

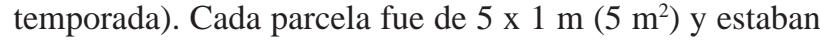
separadas entre sí por al menos 50 metros y fueron distribuidas tratando de abarcar la mayor variabilidad en composición florística y estructura de la vegetación de cada sitio de estudio. Debido a que se observó que los senderos eran ambientes muy utilizados para el tránsito de animales dispersores se estableció parte de las parcelas en las zonas aledañas a aquellos senderos más marcados o con mayores señales de uso por animales. Así se establecieron 10 de las 24 parcelas en los senderos, ubicándose el resto en el interior del bosque. En cada cuadrata se contaron el número de plántulas de ambas especies focales.

Análisis estadísticos. Para determinar diferencias estadísticas en el número de frutos producidos por individuo entre G. triacanthos y A. aroma, se realizaron para las temporadas 2008 y 2009 ANDEVA en bloques, tomando a la especie como factor fijo y al sitio como factor aleatorio (bloque). Para cumplir los supuestos de normalidad y homogeneidad de varianzas necesarios para realizar el análisis, se trabajó con la variable transformada a logaritmo decimal del número de frutos. Para la producción de frutos de la temporada 2010, el banco de semillas, y la densidad de plántulas no se cumplieron los supuestos, aun transformando las variables. Por esta razón se realizaron modelos lineales generalizados mixtos (GLMMs) con distribución de Poisson. La elección del modelo que mejor se ajustó a los datos fue determinada mediante comparación de modelos utilizando la prueba $\mathrm{c}^{2}$ de la función Imer del paquete lme4. En caso de existir sobre-dispersión en el modelo escogido se utilizó la función glmmPQL del paquete MASS. La función glmmPQL no permite la comparación entre modelos, por lo cual la elección del modelo que mejor se ajustó a los datos fue en todos los casos utilizando la prueba c ${ }^{2}$ (Valfré-Giorello et al. 2012 y referencias allí citadas). Los parámetros de los modelos fueron estimados utilizando la aproximación de Laplace y máxima verosimilitud en el caso de la función Lmer y glmmPQL, respectivamente. Para las variables producción de frutos de la temporada 2010, banco de semillas y densidad de plántulas alrededor de adultos de cada especie, se utilizó la especie como efecto fijo y el sitio como aleatorio. Para la densidad de plántulas en los sitios se utilizó el mismo efecto fijo pero como efecto aleatorio se utilizó la parcela. Los ANDEVA se realizaron con el programa SPSS y los GLMMs con R (R Development Core Team 2012).

\section{RESULTADOS}

Producción de frutos. En las temporadas 2008 y 2009, la especie exótica $G$. triacanthos, produjo mayor cantidad de frutos por individuo que A. aroma $\left(F_{1,38}=8,06, P=0,007\right.$; $F_{1,38}=35,51, P<0,001$, para la temporada 2008 y 2009, respectivamente) (figura 1). En la temporada 2010 ambas especies produjeron una muy baja cantidad de frutos, no encontrándose diferencias significativas entre las especies $(\mathrm{t}=1,38, P=0,18)$ (figura 1 ). 


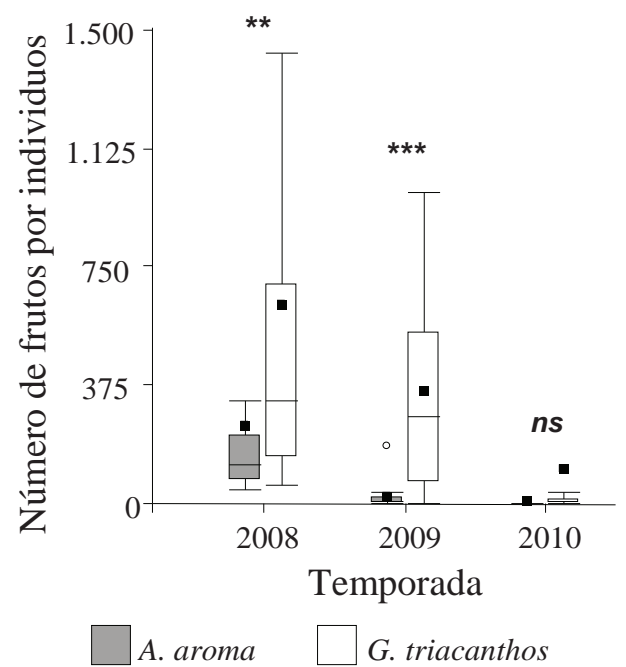

Figura 1. Número de frutos por individuo para la especie nativa A. aroma y la exótica invasora $G$. triacanthos en las temporadas reproductivas 2008,2009 y $2010 . * * P<0,01, * * * P<0,001$, ns $=$ no significativo.

Number of fruits per individual for the native species A. aro$m a$ and the exotic invasive $G$. triacanthos in 2008, 2009 and 2010 reproductive seasons. $* * P<0.01$, $* * * P<0.001, \mathrm{~ns}=$ not significant.

Banco de semillas. Se encontraron semillas de ambas especies en el suelo inmediatamente después de terminada la dispersión y comenzadas las primeras lluvias ("banco de semillas luego de la dispersión”), y antes del nuevo periodo de dispersión ("banco de semillas previo al nuevo periodo de dispersión”). El número total de semillas viables por $\mathrm{m}^{2}$ encontradas en el "banco de semillas luego de la dispersión" fue significativamente mayor para $G$. triacanthos en las dos temporadas analizadas $(\mathrm{t}=3,47, P=0,001$; $\mathrm{t}=3,05, P=0,004,2008$ y 2009 , respectivamente) (figura 2A). El mismo patrón se observó para el número total de semillas viables por $\mathrm{m}^{2}$ encontradas en el "Banco de semillas previo al nuevo periodo de dispersión” ( $\mathrm{t}=3,43$, $P=0,002, t=2,84, P=0,007$, para las temporadas $2008 \mathrm{y}$ 2009 , respectivamente) (figura $2 \mathrm{~B}$ ).

Plántulas alrededor de los adultos de cada especie y en los sitios. El número de plántulas por $\mathrm{m}^{2}$ alrededor de los adultos fue mayor para $G$. triacanthos respecto a $A$. aroma en las dos temporadas de muestreo $(t=3,39 P<0,001$; $z=3,04, P=0,002$, temporadas 2008 y 2009, respectivamente) (figura 3 ). Si bien en promedio G. triacanthos presentó un mayor número de plántulas por $\mathrm{m}^{2}$ en las cuadratas de los sitios de estudio ( $\overline{\mathrm{X}}=0,34 \pm 0,15$ y $\overline{\mathrm{X}}=0,05$ $\pm 0,13$ para $G$. triacanthos y A. aroma temporada 2008 y $\overline{\mathrm{x}}=0,15 \pm 0,77$ y $\overline{\mathrm{x}}=0,07 \pm 0,14$ para $G$. triacanthos $\mathrm{y}$ A. aroma temporada 2009), la influencia del factor especie no resultó estadísticamente significativa en ninguna de las temporadas $\left(\mathrm{c}^{2}=2,55 \times 10^{-8}, P=0,99 ; \mathrm{c}^{2}=2,5 P=0,11\right.$ temporadas 2008 y 2009, respectivamente).

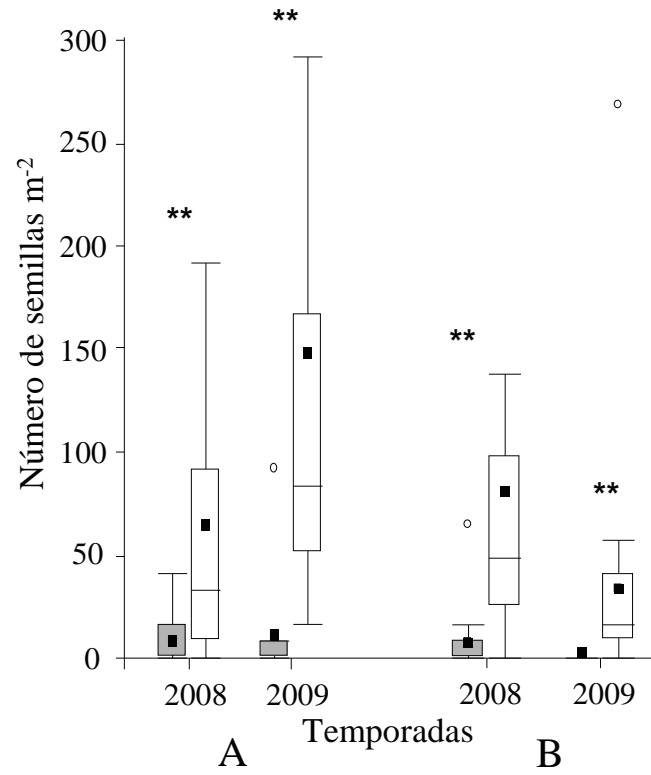

\section{A. aroma $\square$ G. triacanthos}

Figura 2. Número de semillas por $\mathrm{m}^{2}$ para la especie nativa A. aroma y parala exótica invasora G. triacanthos enlas temporadas 2008 y 2009. A) Banco de semillas luego de la dispersión. B) Banco de semillas previo al nuevo periodo de dispersión. $* * P<0,01$

Number of seeds per $\mathrm{m}^{2}$ for the native species A. aroma and the exotic invasive G. triacanthos in 2008 and 2009 seasons. A) Seed banks after fruit dispersal. B) Seed bank before the new period of dispersal. $* * P<0.01$.

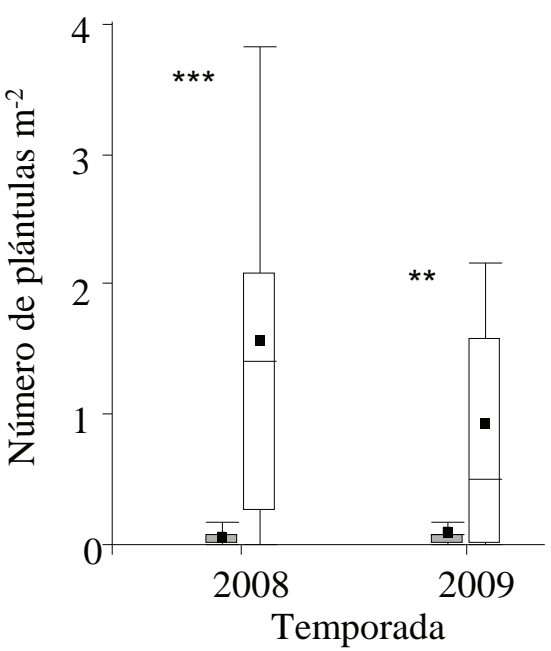

\section{A. aroma \\ G. triacanthos}

Figura 3. Número de plántulas por $\mathrm{m}^{2}$ alrededor de los individuos de la especie nativa $A$. aroma y la exótica invasora $G$. triacanthos en las temporadas 2008 y 2009. ** $P<0,01$, $* * * P<0,001$.

Number of seedlings per $\mathrm{m}^{2}$ for the native species A. aro$m a$ and the exotic invasive G. triacanthos in 2008 and 2009 seasons. ${ }^{* *} P<0.01,{ }^{* * *} P<0.001$ 


\section{DISCUSIÓN}

Los estudios comparativos entre plantas exóticas invasoras y nativas incluyendo más de una temporada reproductiva son poco frecuentes y menos aun considerando más de una variable del ciclo reproductivo (Martin y Canham 2010). En este estudio, la especie exótica invasora Gleditsia triacanthos presentó en las distintas temporadas similares tendencias en relación a la nativa Acacia aroma, las cuales consistieron en una mayor producción frutos, mayor densidad de semillas en el banco y de plántulas alrededor de los adultos en la especie exótica, sin presentar diferencias en relación a las plántulas en los sitios. Si bien se pueden observar variaciones en cada una de las especies entre temporadas, las mismas no parecen incidir en la significancia de las diferencias entre las especies.

La presencia de variaciones en la producción de frutos es un proceso común en especies leñosas (Kelly y Sork 2002, Pairon et al. 2006). En este estudio la especie exótica presentó una mayor producción de frutos en ambas temporadas estudiadas. Por otro lado, la especie nativa presentó una baja producción de frutos en la temporada 2009 y 2010, en esta última también la especie exótica presentó una baja producción de frutos. En consecuencia, se podría sugerir que las dos especies presentan un patrón similar, siendo la temporada 2008 la de mayor producción para ambas. Posteriormente, presentan una caída en la producción de frutos en la temporada 2009, que conduce a una drástica reducción en la reproducción de la especie nativa, y una nueva caída en la temporada 2010, que ocasiona una muy baja producción de frutos en ambas especies, totalizando así dos temporadas con una muy baja producción de frutos en la especie nativa (figura 1). Sin bien, podrían existir una multiplicidad de factores que causen estas fluctuaciones, dado que estas especies tienen similar fenología reproductiva, es posible que algún factor climático este determinando este patrón (por ejemplo el nivel de precipitaciones podría influir en la cantidad de flores producidas o en la abundancia de polinizadores, Kelly y Sork 2002). Cabe destacar que, si consideramos los datos obtenidos previamente (Ferreras y Galetto 2010), la especie exótica y la nativa no presentaron diferencias significativas en la temporada 2007. Por tanto, no se presentaría el patrón conjunto aquí destacado, ya que comparando las temporadas 2007 y 2008 si bien G. triacanthos aumenta en promedio su producción en la temporada 2008, A. aroma la reduce, sugiriendo que existiría un factor que afectaría diferencialmente a cada una. Sin embargo, es pertinente destacar que el estudio de la temporada 2007 presenta una escala espacial más reducida. Asimismo, dada la mayor cantidad de semillas por fruto, en dicho trabajo previo (Ferreras y Galetto 2010) la especie exótica sí superó a la nativa en la producción total de semillas; lo cual sumado a los resultados aquí encontrados indicaría, al menos en relación a la nativa analizada, que la especie exótica tendría la potencialidad de aumentar fácilmente su densidad y ampliar su rango de distribución. Por último, si bien en este estudio la especie exótica presentó variaciones en la cantidad de frutos producidos en las distintas temporadas, cabe resaltar que la especie nativa presentaría mayor cantidad de años con fallas o reducción en la producción de frutos, implicando baja producción de frutos durante años consecutivos, en relación a dicha especie exótica. Este mismo patrón fue encontrado por Martin y Canham (2010) para otras especies exóticas y nativas. Asimismo, estos autores también advierten que es importante considerar que el lapso de tiempo evaluado sería muy corto para los tiempos en que pueden ocurrir estas fluctuaciones, por lo cual no se puede descartar que la especie exótica presente también una sucesión de años de baja producción de frutos. Sin embargo, por observaciones personales en el área de estudio durante años sucesivos a este estudio esto no ocurriría comúnmente en G. triacanthos.

Similar a lo ocurrido con la comparación entre especies para la producción de frutos, las tendencias en la densidad del banco de semillas también presentaron un patrón coincidente entre temporadas, siendo mayor la densidad en la especie exótica tanto en el "banco de semillas luego de la dispersión" como en el "banco de semillas previo al nuevo periodo de dispersión”. Lo mismo ocurrió en la temporada 2007, aunque en uno de los muestreos dichas diferencias no fueron estadísticamente significativas (Ferreras y Galetto 2010). Las diferencias aquí encontradas podrían estar altamente influenciadas por valores dispares en la producción frutos (particularmente importante en este trabajo debido al diseño desarrollado -i.e., muestreo de banco en cada uno de los individuos focales) y podrían estar indicando que ningún otro factor que afecte la variabilidad de la densidad de semillas del banco - e.g., factores abióticos (e.g., lluvias), densidad de depredadores post-dispersivos, etc. - estaría contrarrestando significativamente ese patrón. El banco de semillas es muy importante para el mantenimiento de las poblaciones sujetas a disturbios y permite, asimismo, el mantenimiento de la variabilidad genética (Baskin y Baskin 1998). Por tanto, la mayor densidad de semillas que se observa en el banco de la especie exótica podría favorecer el mantenimiento de sus poblaciones en las comunidades donde se introduce. Asimismo, estos resultados sugerirían que aún en años donde la especie exótica no produzca frutos, el reclutamiento de un cierto número de individuos podría estar asegurado desde las semillas del banco.

Finalmente, la densidad de plántulas (tanto alrededor de los adultos, como en el área de estudio) presentó el mismo patrón entre ambas especies para las dos temporadas analizadas, al igual que en las variables previamente discutidas. Dichos resultados también concuerdan con lo hallado previamente (Ferreras y Galetto 2010). Alrededor de los adultos la densidad fue mayor en la especie exótica en ambas temporadas. Sin embargo, resulta destacable que las diferencias entre las especies fueron mayores en la temporada 2008 en relación a la 2009. La variabilidad en 
la densidad de plántulas ha sido comúnmente observada y puede estar influenciada por la variabilidad en la producción de frutos, la depredación pre- y post-dispersiva, factores climáticos, etc. (Chaneton et al. 2004). Definir cuál de estas variables sería responsable de generar el patrón encontrado requeriría un análisis más detallado. En las parcelas distribuidas en los sitios no se encontraron los resultados esperados, dado que no se observaron diferencias significativas entre las especies, y la densidad de plántulas registrada en ambas especies, en las dos temporadas, fue baja en relación a la registrada alrededor de los individuos focales. Así, la mayor densidad de plántulas de estas especies estaría alrededor de los adultos, lo cual concuerda con el patrón agregado de crecimiento que se suele observar en ambas especies y es un aspecto notable en la especie exótica ya que la misma forma bosques mono-específicos en estados de invasión avanzados. Por otro lado, en la especie exótica el reclutamiento en los sitios, aunque sin presentar diferencias con la especie nativa y siendo de menor densidad que alrededor de los individuos focales, tendría mucha importancia ya que desde allí se podrían constituir nuevos focos de invasión.

$\mathrm{Al}$ igual que se observó previamente (Ferreras y Galetto 2010), los resultados aquí obtenidos indican que G. triacanthos presenta características que favorecerían su alta capacidad invasión. Además, estos resultados fueron consistentes entre temporadas y sugerirían una rápida velocidad de invasión y podrían explicar la amplia distribución que actualmente registra en distintos lugares de Argentina (de Viana y Colombo Speroni 2000, Chaneton et al. 2004). Cabe resaltar que sin bien este estudio intenta ampliar la escala temporal, sólo considera dos o tres temporadas reproductivas y sería muy importante poder hacer este tipo de evaluaciones durante más temporadas y confirmar o descartar los patrones encontrados. Además, sería importante comparar esta especie exótica con otras especies nativas arbóreas de importancia en el área de estudio (por ejemplo, Prosopis alba), para determinar, con mayor precisión aquellas características que estarían involucradas en su expansión. Por último, en lo referido a posibles estrategias de manejo, es importante resaltar la presencia de un abundante banco de semillas en G. triacanthos, registrado en las dos temporadas evaluadas, lo cual sugiere que cualquier estrategia que se desarrolle debe implicar un monitoreo del área manejada para evitar el reclutamiento de nuevos individuos. Los mismos deberían realizarse por periodos prolongados de tiempo dado que la mayoría de las semillas presentes en el banco necesitaron escarificarse para germinar, sumado a que las semillas de esta especie tendrían una alta longevidad (Ferreras et al. 2014). Asimismo, la mayor densidad de plántulas en relación a A. aroma (al menos alrededor de los adultos), en ambas temporadas, sugeriría que esta especie podría aumentar rápidamente sus poblaciones, por lo cual mientras más tempranas sean las estrategias de manejo es posible que estas sean más exitosas. Dadas las características hasta ahora registradas por esta especie exótica invasora sería necesario seguir ampliando la información para establecer claras estrategias de manejo que favorezcan la conservación del bosque chaqueño serrano.

\section{AGRADECIMIENTOS}

Agradecemos a los dueños de los campos y a la comunidad de la Rancherita por facilitar la investigación en las áreas de estudio. A dos revisores anónimos y el editor por los valiosos comentarios realizados a versiones previas de este manuscrito. Al Consejo Nacional de Investigaciones Científicas y Técnicas (CONICET), SECyT (UNC) y la Agencia Nacional de Promoción Científica y Tecnológica por el apoyo financiero. A.E.F. es becaria post-doctoral de CONICET y G.F. y L.G son investigadores de la misma institución.

\section{REFERENCIAS}

Baskin CC, JM Baskin. 1998. Seeds: Ecology, biogeography and evolution of dormancy and germination. San Diego, EUA. Academic Press. 666 p.

Chaneton EJ, NC Mazia, M Machera, A Uchitel, CM Ghersa. 2004. Establishment of Honey Locust (Gleditsia triacanthos) in burned Pampean Grassland. Weed Technology 18(1): 1325-1329. DOI: 10.1614/0890-037X(2004)018[13 25:EOHLGT]2.0.CO;2

Cordell S, R Ostertag, B Rowe, L Sweinhart, L Vasquez-Radonic, J Michaud, T Colleen Cole, JR Schulten. 2009. Evaluating barriers to native seedling establishment in an invaded Hawaiian lowland wet forest. Biological Conservation 142(12): 2997-3004. DOI: 10.1016/j.biocon.2009.07.033.

De Fina AL. 1992. Aptitud agroclimática de la república Argentina. Buenos Aires, Argentina. Academia Nacional de Agronomía y Veterinaria. 402 p.

de Viana ML, F Colombo Speroni. 2000. Invasión de Gleditsia triacanthos L. (Fabaceae) en el Bosque de San Lorenzo, Salta, Argentina. In Grau HR, MR Aragón eds. Ecología de árboles invasores de las yungas Argentinas. Tucumán, Argentina. LIEY-UTN. p. 71-84.

Ebinger JE, DS Seigler, HD Clarke. 2000. Taxonomic revision of South American species of the genus Acacia subgenus Acacia (Fabaceae: Mimosoideae). Systematic Botany 25(4): 588-617. DOI: 10.2307/2666723.

Ferreras AE, L Galetto. 2010. From seed production to seedling establishment: Important steps in an invasive process. Acta Oecologica 36(2): 211-218. DOI: 10.1016/j. actao.2009.12.005.

Ferreras AE, G Funes, L Galetto. 2014. The role of seed germination in the invasive process of Honey locust (Gleditsia triacanthos L., Fabaceae): comparison with a native confamilial. Plant Species Biology. DOI: 10.1111/1442-1984.12041

Fonseca CR, DL Guadagnin, C Emer, S Masciadri, P Germain, SM Zalba. 2013. Invasive alien plants in Pampas grasslands: a tri-national cooperation challenge. Biological invasions 15(8): 1751-1763. DOI: 10.1007/s10530-013-0406-2.

Giorgis MA, AM Cingolani, F Chiarini, J Chiapella, G Barboza, L Ariza Espinar, R Morero, D Gurvich, PA Tecco, R Su- 
bils, M Cabido. 2011. Composición florística del Bosque Chaqueño Serrano de la Provincia de Córdoba, Argentina. Kurtziana 36(1): 9-43.

Hastings A, K Cuddington, KF Davies, CJ Dugaw, S Elmendorf, A Freestone, S Harrison, M Holland, J Lambrinos, U Malvadkar, BA Melbourne, K Moore, C Taylor, D Thomson. 2005. The spatial spread of invasions: new developments in theory and evidence. Ecology Letters 8(1): 91-101. DOI: 10.1111/j.1461-0248.2004.00687.x.

Kelly D, VL Sork. 2002. Mast seeding in perennials plants: Why, how, where? Annual Review of Ecology and Systematic 33: 427-447. DOI: 10.1146/annurev.ecolsys.33.020602.095433.

Krinke L, L Moravcová, P Pyšek, V Jarošik, J Pergl, I Pergolvá. 2005. Seed bank of an invasive alien, Heracleum mantegazzianum, and its seasonal dynamics. Seed Science Research 15(3): 239-248. DOI: 10.1079/SSR2005214.

Maguire LA. 2004. What can decision analysis do for invasive species management? Risk Analysis 24(4): 859-868. DOI: 10.1111/j.0272-4332.2004.00484.x.

Martin PH, CD Canham. 2010. Dispersal and recruitment limitation in invasive versus exotic tree species: life-history strategies and Janzen-Connell effects. Oikos 119(5): 807-824. DOI: 10.1111/j.1600-0706.2009.17941.x

Pairon M, O Chabrerie, C Mainer Casado, A-L Jacquemart. 2006. Sexual regeneration traits linked to black cherry (Prunus serotina Ehrh.) invasiveness. Acta Oecologica 30(2): 238247. DOI: 10.1016/j.actao.2006.05.002.

Pyšek P, DM Richardson. 2007. Traits associated with invasiveness in alien plants: where do we stand? In Nentwig W ed. Biological Invasions. Berlin, Alemania. Springer-Verlag. p. 97-125.

R Develoment Core Team. 2012. R: A language environment for statistical computing. R Foundation for Statistical Computing. Vienna, Austria. ISBN 3-900051-07-0. Disponible en: http://www.rproject.org.
Richardson DM, M Rejmánek. 2004. Conifers as invasive aliens: a global survey and predictive framework. Diversity and Distributions 10(5-6): 321-331. DOI: 10.1111/j.13669516.2004.00096.x.

Richardson DM, M Rejmánek. 2011. Trees and shrubs as invasive alien species-a global review. Diversity and Distributions 17(5): 788-809. DOI: 10.1111/j.1472-4642.2011.00782.x.

Rudgers JA, JD Hoeksema. 2003. Inter-annual variation in above and belowground herbivory on a native, annual legume. Plant Ecology 169(1): 105-120. DOI: 10.1023/A:1026221602968.

Sakai AK, FW Allendorf, JS Holt, DM Lodge, J Molofsky, KA With, S Bauhman, RJ Cabin, JE Cohen, NC Ellstrand, DE McCauley, P O’Neil, IN Parker, JN Thompson, SG Weller. 2001. The population biology of invasive species. Annual Review of Ecology, Evolution and Systematics 32:305-332. DOI: 10.1146/annurev.ecolsys.32.081501.114037.

Strauss SY, CO Webb, N Salamin. 2006. Exotic taxa less related to native species are more invasive. Proceedings of National Academy of Science 103(15): 5841-5845. DOI:10.1073/ pnas.0508073103.

Valfré-Giorello TA, L Ashworth, D Renison. 2012 Patrones de germinación de semillas de Sebastiania commersoniana (Baillon) Smith et Downs (Euphoriaceae), árbol nativo del Chaco Serrano de interés en restauración. Ecología Austral 22(2):92-100.

Vilà M, C Basnou, P Pyšek, M Josefsson, P Genovesi, S Gollasch, W Nentwig, S Olenin, A Roques, D Roy, PE Hulme, DAISE partners. 2010. How well do we understand the impacts of alien species on ecosystem services? A pan-European cross-taxa assessment. Fronts in Ecology and Environment 8(3): 135-144. DOI: 10.1890/080083.

Zalba SM, CB Villamil. 2002. Invasion of woody plants in relictual native grasslands. Biological invasions 4(1-2): 55-72. DOI: 10.1023/A:1020532609792. 